\title{
Kaposi's sarcoma complicating long-term corticotherapy for rheumatoid arthritis
}

\section{Sara Elloudi, Hakima Elmahi, Salim Gallouj, Fatima Zahra Mernissi}

\author{
Department of Dermatology and Venereology, University Hospital Hassan II Fez, Fez, Morocco
}

Corresponding author: Dr. Sara Elloudi, E-mail: saraelloudi@gmail.com

\begin{abstract}
Kaposi's sarcoma (KS) is a lymphoangioproliferative neoplasm induced by human herpes virus 8 (HHV-8). KS caused by iatrogenic immunosuppression is rare. Iatrogenic KS can present a therapeutic dilemma, as decreasing the level of immunosuppression may not be possible or may come at the high cost of organ rejection, such as in the case of organ transplant recipients or organ damage in the case of patients with autoimmune disorders. We report a new case of Kaposi's sarcoma induced by corticosteroids.
\end{abstract}

Key words: Kaposi; Corticosteroids; Rheumatoid arthritis

\section{INTRODUCTION}

Kaposi's sarcoma (KS) is a lymphoangioproliferative neoplasm induced by human herpes virus 8 (HHV-8). Four clinical variants have been recognized: Classical, African endemic, AIDS-related, and KS caused by iatrogenic immunosuppression. The latter is typically associated with the use of immunosuppressive therapy in organ transplant recipients; however, iatrogenic KS can also occur in patients receiving corticosteroids for other indications, such as autoimmune disorders. We describe a 44-year-old woman suffering from rheumatoid arthritis treated with low doses of prednisone who developed Kaposi's sarcoma (KS).

\section{CASE REPORT}

A 44-year-old woman suffering from rheumatoid arthritis for 30 years treated with low doses of prednisone who developed erythematous patchs and nodules purplish with smooth and keratosis surface, of varying size interesting feet, legs, hand and forearm, without mucosal lesions (Fig. 1). The dermoscopy showed a rainbow pattern, Homogeneous blue-red color and squamous surface (Fig. 2). This patient was not affected with AIDS. Skin biopsy confirmed plaque-stage $\mathrm{KS}$ and imaging ruled out systemic involvement. Diagnosis of cutaneous iatrogenic KS was made, corticosteroid was gradually stopped and replaced by methotrexate to treat rheumatoid arthritis. The lesions regressed then relapsed. we opted for surgical excision of nodules with radiotherapy for lesions of the feet and legs with improvement.

\section{DISCUSSION}

Kaposi's sarcoma has been reported in patients receiving immunosuppressive therapy, most of whom are organ transplant recipients. The development of Kaposi's sarcoma after treatment with corticosteroids has been reported in only 38 patients who have not had acquired immunodeficiency syndrome or undergone organ transplantation [1]. Data from the literature clearly show that corticosteroid therapy may trigger the development of Kaposi's sarcoma in patients who usually possess several other pathogenetic factors of that disease, such as pre-existing immune deficiency, environmental (viruses) or genetic factors [2]. In the literature only 8 rheumatoid arthritis patients who developed KS during steroid therapy have been reported [3]. In some cases, withdrawing corticosteroids

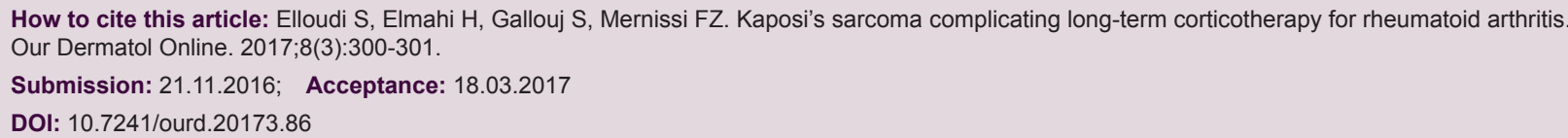




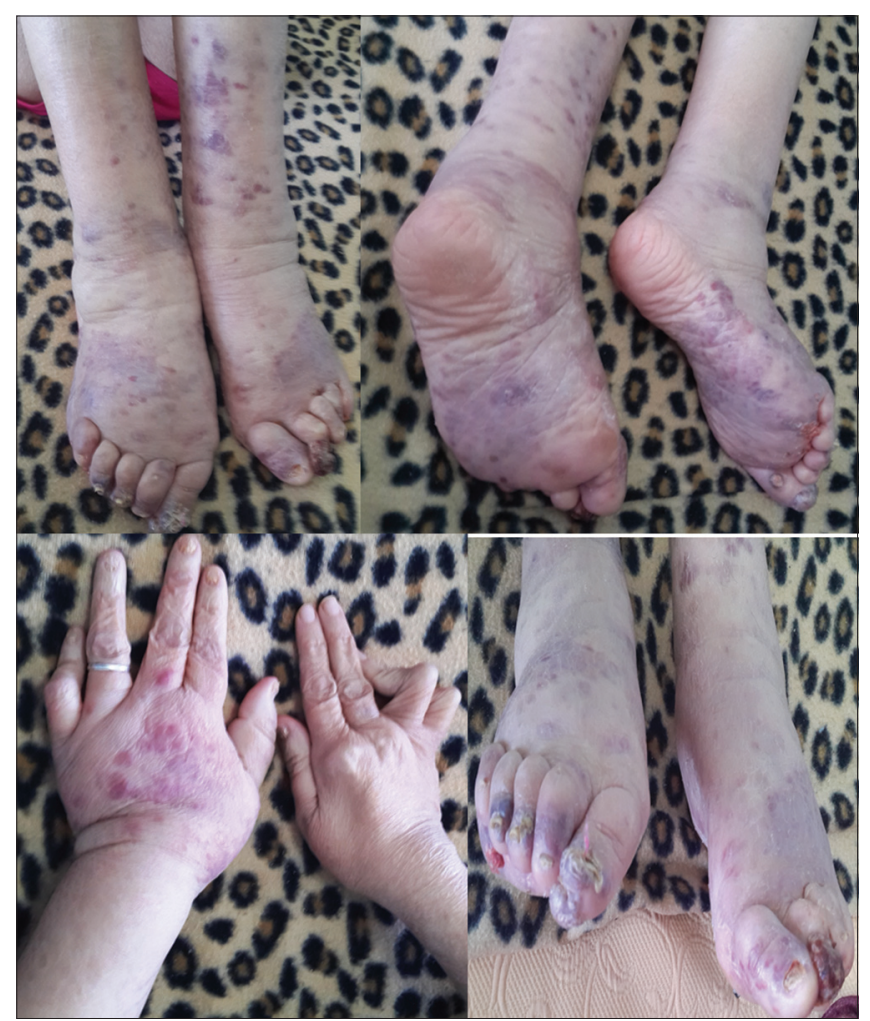

Figure 1: Erythematous patchs and nodules purplish at the extremities.

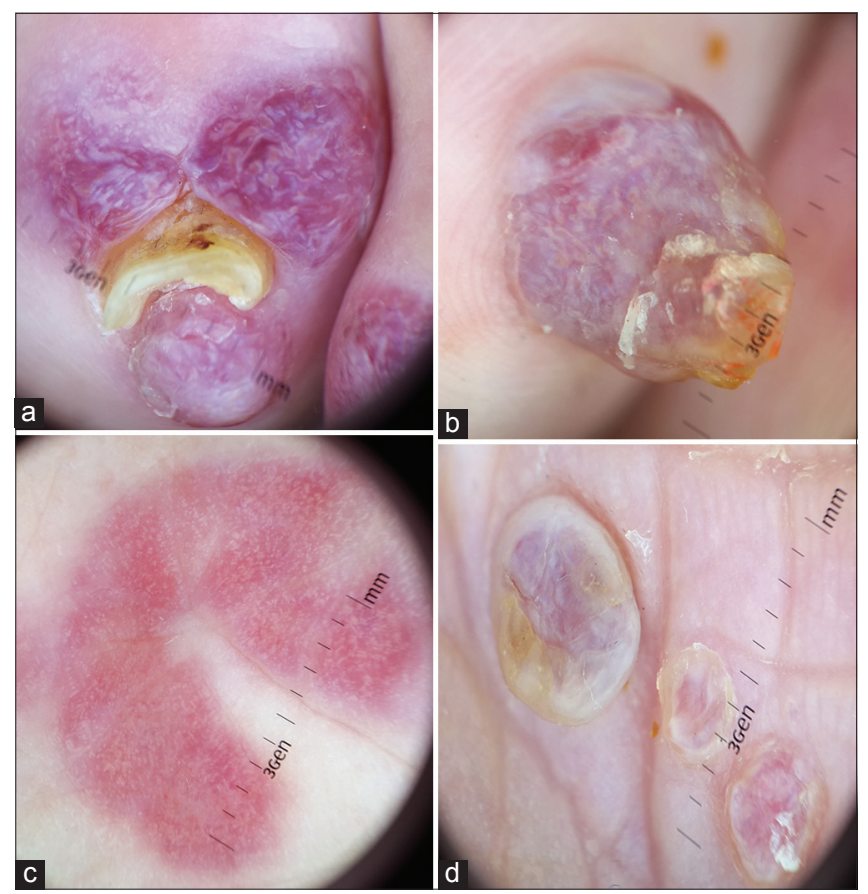

Figure 2: Dermoscopic patterns of different lesions. ( $a$ and b) Rainbow pattern, (c) Homogeneous red color, (d) Squamous surface. may result in complete remission of the cutaneous lesions [4]. In-vitro studies suggest corticosteroids induce $\mathrm{KS}$ by indirectly inhibiting transforming growth factor beta, a protein that inhibits growth of endothelial cells [5]. In our patient, The lesions regressed after discontinuation of corticosteroid therapy but reappeared with methotrexate, prompting alternative treatment.

\section{CONCLUSION}

Maintaining immunosuppression leads to further progression of iatrogenic KS. However, restoring immune defenses by reducing or withdrawing immunosuppression may induce spontaneous KS remission and represents the first-line therapy for iatrogenic KS.Iatrogenic KS can present a therapeutic dilemma, as decreasing the level of immunosuppression may not be possible or may come at the high cost of organ rejection, such as in the case of organ transplant recipients or organ damage in the case of patients with autoimmune disorders. This case highlights the therapeutic challenge of iatrogenic KS, as reduction or removal of immunosuppression may not always lead to resolution of KS. In these cases or in cases in which reduction of immunosuppression may not be possible, other therapy such as surgery or radiotherapy should be considered.

\section{REFERENCES}

1. Trattner A, Hodak E, David M, Neeman A, Sandbank M. Kaposi's sarcoma with visceral involvement after intraarticular and epidural injections of corticosteroids. J Am Acad Dermatol. 1993;29:890-4.

2. Bruet A, Mahe A, Sei JF, Mathe C, Felsenheld C, Lechevalier L, et al. Kaposi's sarcoma complicating long-term corticotherapy for severe asthma. Rev Med Interne. 1990;11:322-4.

3. Casoli P, Tumiati B. Rheumatoid arthritis, corticosteroid therapy and Kaposi's sarcoma: A coincidence? A case and review of literature. Clin Rheumatol. 1992;11:432-5.

4. Antman K, Chang Y. Kaposi's sarcoma. N Engl J Med. 2000;342:1027-38.

5. Cai J, Zheng T, Lotz M, Zhang Y, Masood R, Gill P. Glucocorticoids induce Kaposi's sarcoma cell proliferation through the regulation of transforming growth factor-beta. Blood. 1997;89:1491-500.

Copyright by Sara Elloudi, et al. This is an open access article distributed under the terms of the Creative Commons Attribution License, which permits unrestricted use, distribution, and reproduction in any medium, provided the original author and source are credited.

Source of Support: Nil, Conflict of Interest: None declared. 